\title{
Langmuir Monolayers of Co Nanoparticles and Their Patterning by Micro-contact Printing
}

\author{
Jong-Il Park, ${ }^{\dagger}$ Woo-Ram Lee, ${ }^{\ddagger}$ Sung-Soo Bae, ${ }^{\dagger}$ Youn Joong Kim, ${ }^{\S}$ Kyung-Hwa Yoo, Jinwoo Cheon ${ }^{*},{ }^{\ddagger}$ and Sehun Kim ${ }^{*}, \dagger$
}

Contribution from the Department of Chemistry, Korea Advanced Institute of Science and Technology, Daejeon 305-701, Korea, Department of Chemistry, Yonsei University, Seoul, 120-749, Korea, Department of Physics, Yonsei University, Seoul, 120-749, Korea, Division of Nano-Material \& Environmental Science, Korea Basic Science Institute, Daejeon, 305-333, Korea

\section{Supporting Information}

As-prepared Co nanoparticles and their LB films before and after annealing were analyzed by X-ray photoelectron spectroscopy (XPS) and X-ray absorption spectroscopy (XAS).

XPS spectra for the as-prepared Co nanoparticles show Co $2 \mathrm{p}_{3 / 2}$ and $2 \mathrm{p}_{1 / 2}$ peaks at binding energy of 779.4 and $795.4 \mathrm{ev}$, respectively. Broadening by surface oxidation was observed at as-prepared samples because Co nanoparticles were stabilized by tetradecanoic acid (Fig. S1A-(a)). These peaks for the LB film of Co nanoparticles shift to781.1 and 796.7ev that correspond to the valence state of $\mathrm{Co}^{2+}$, indicating that the Co nanoparticles are oxidized in basic solution and ambient condition (Fig. S1A-(b)). Oxidized Co nanoparticles are reduced by heating the Co nanoparticles under $\mathrm{H}_{2}$ at $300^{\circ} \mathrm{C}$. The XPS spectra recorded for Co nanoparticles after the reduction process show peaks at 777.5 and 792.7ev in Figure S1A-(c), but oxide peaks still present in the samples due to the re-oxidation that occurs very easily during a XPS measuring process because stabilizer removed Co nanoparticles were directly contacted with air expose to ambient condition. To avoid re-oxidation problem, each sample was sealed in Kapton tape and analyzed by XAS. As shown in figure $\mathrm{S} 1 \mathrm{~B}$, peak $\mathrm{P}_{1}$ for initial Co nanoparticles is shifted to a lower energy after LB filming process by oxidation and returned to the pure metallic Co state after the reduction process under $5 \% \mathrm{H}_{2}$. In conclusion, Co nanoparticles are oxidized during LB filming procedure and reduced by annealing at $300^{\circ} \mathrm{C}$ under $\mathrm{H}_{2}$ gas. 

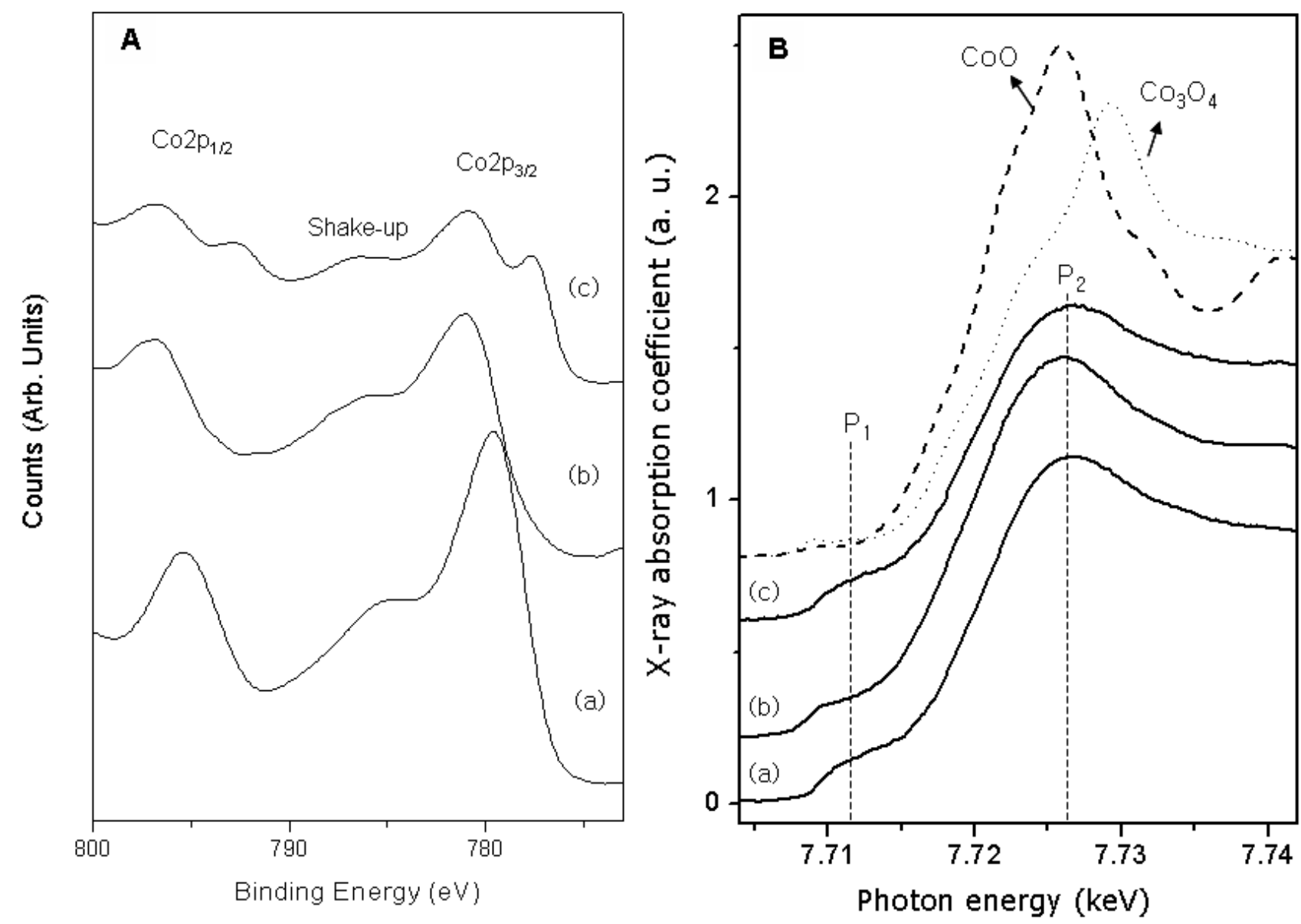

Figure S1. (A) Co 2p XPS spectra and (B) Co K-edge XANES spectra of Co nanoparticles.

(a) initial Co nanoparticles (b) LB films of Co nanoparticles (c) after annealing at $300{ }^{\circ} \mathrm{C}$. 


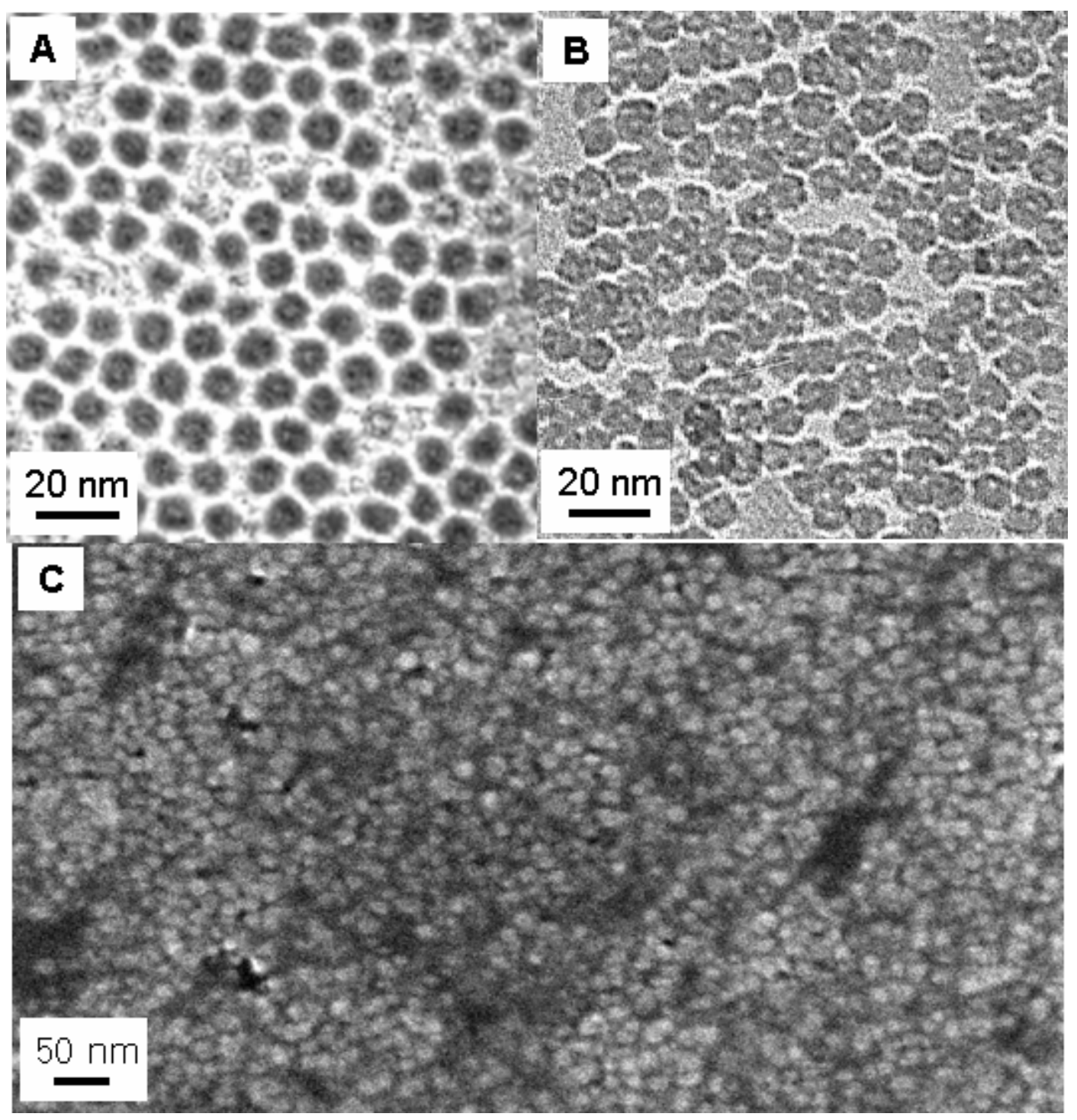

Figure S2. (A) Enlarged TEM images of initial LB monolayer of Co nanoparticles. (B) TEM image and (C) FE-SEM image of LB monolayer after annealing at $300{ }^{\circ} \mathrm{C}$. 